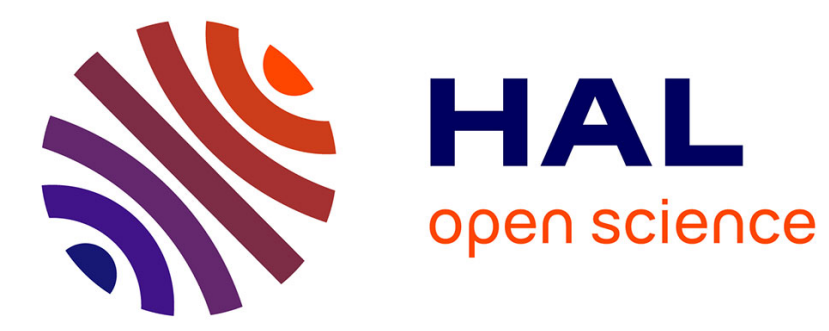

\title{
Effets d'épaisseur et de polarisation dans les spectres d'absorption Mössbauer; essai d'une formule simple
}

\author{
M. Henry, J. Teillet, F. Varret
}

\section{To cite this version:}

M. Henry, J. Teillet, F. Varret. Effets d'épaisseur et de polarisation dans les spectres d'absorption Mössbauer; essai d'une formule simple. Revue de Physique Appliquée, 1980, 15 (6), pp.1095-1102. 10.1051/rphysap:019800015060109500 . jpa-00244827

\section{HAL Id: jpa-00244827 https://hal.science/jpa-00244827}

Submitted on 1 Jan 1980

HAL is a multi-disciplinary open access archive for the deposit and dissemination of scientific research documents, whether they are published or not. The documents may come from teaching and research institutions in France or abroad, or from public or private research centers.
L'archive ouverte pluridisciplinaire HAL, est destinée au dépôt et à la diffusion de documents scientifiques de niveau recherche, publiés ou non, émanant des établissements d'enseignement et de recherche français ou étrangers, des laboratoires publics ou privés. 


\title{
Effets d'épaisseur et de polarisation dans les spectres d'absorption Mössbauer ; essai d'une formule simple
}

\author{
M. Henry, J. Teillet et F. Varret \\ E.R.A. ${ }^{\circ}$ 682, Faculté des Sciences, 72017 Le Mans Cedex, France
}

\begin{abstract}
Résumé. - Nous donnons une expression approchée, simple, de l'intensité des raies, valable pour un absorbeur à raies bien résolues, dont on discute les conditions d'application. Nous analysons les résultats expérimentaux obtenus avec des absorbeurs de fer, soit isotropes, soit aimantés. Par ailleurs, des considérations sur les états propres de polarisation sont développées.
\end{abstract}

\begin{abstract}
We give an extremely simple expression for the line intensities of a multiple line absorber. The expression holds in the case of well resolved lines whose effective thickness is not too large : it is used for experiments performed using iron absorbers, either as powders, or as a foil completely magnetized. The relevantness of the method is discussed. Some insight of the polarization eigenstates is given.
\end{abstract}

Introduction. - Le profil de la raie d'absorption Mössbauer se calcule par une intégrale [1], dite intégrale de transmission, dont l'expression dans le cas d'un absorbeur à raie unique, et en l'absence d'effets d'auto-absorption, est donnée ci-dessous :

$$
\begin{aligned}
I\left(E_{\mathrm{s}}\right) \sim \int_{-\infty}^{+\infty} & \frac{\Gamma_{\mathrm{s}} / 2 \Pi}{\left(E-E_{\mathrm{s}}\right)^{2}+\Gamma_{\mathrm{s}}^{2} / 4} \times \\
& \times\left[1-\exp \left(-\frac{T \Gamma_{0}^{2} / 4}{\left(E-E_{0}\right)^{2}+\Gamma_{0}^{2} / 4}\right)\right] \mathrm{d} E
\end{aligned}
$$

avec

$$
T=\text { fan } \sigma_{0} t
$$

où

$E_{\mathrm{s}}, E_{0}=$ énergies des raies de la source et de l'absorbeur,

$\Gamma_{\mathrm{s}}, \Gamma_{0}=$ largeurs des raies de la source et de l'absorbeur,

$T=$ épaisseur effective de l'absorbeur,

$f=$ facteur Debye-Waller de l'absorbeur (fraction résonnante),

$a n=$ nombre de noyaux Mössbauer dans l'absorbeur, par unité de volume,

$\sigma_{0}=$ section efficace du noyau Mössbauer,

$t=$ épaisseur physique de l'échantillon.

L'intensité de la raie Mössbauer, c'est-à-dire la surface de la raie observée dans le spectre est donnée par l'intégrale

$$
A=\int_{-\infty}^{+\infty} I\left(E_{\mathrm{s}}\right) \mathrm{d} E_{\mathrm{s}}
$$

C'est cette dernière formule que Lang [2] a utilisée pour calculer numériquement $A$ en fonction de l'épaisseur effective dans divers cas. Cependant les tabulations de la fonction $A\left(T, \Gamma_{0}, \Gamma_{\mathrm{s}}\right)$ qu'il a données, ou que l'on peut recalculer aisément, ne sont pas utilisées couramment lors de l'ajustement des spectres ; de nombreux auteurs [3] utilisent directement l'intégrale de transmission pour ajuster le profil exact de la raie Mössbauer, et cette procédure demande un temps de calcul (ordinateur) assez long.

Il existe [4a] une expression analytique de l'intégrale $A$, dans le cas où la source et l'absorbeur ont la même largeur de raie :

$$
A \sim T \exp \left(-\frac{T}{2}\right)\left[I_{0}\left(\frac{T}{2}\right)+I_{1}\left(\frac{T}{2}\right)\right]
$$

où $I_{0}$ et $I_{1}$ sont des fonctions de Bessel modifiées de première espèce.

Nous avons récemment proposé [5], pour un problème d'auto-absorption conduisant à une équation très semblable à l'équation (2), d'utiliser une expression approchée de l'équation (2), déjà donnée $[4 b]$ :

$$
A \sim \frac{T}{1+\frac{T}{4}}
$$

Les expressions (2) et (3) ont des développements limités identiques jusqu'aux termes en $T^{3}$ (inclus). 
L'erreur relative sur $A$ introduite par la formule approchée (3) est inférieure à $10^{-2}$ lorsque $T$ est inférieur à 2. La figure 1 montre l'évolution de cette erreur en fonction de $T$.

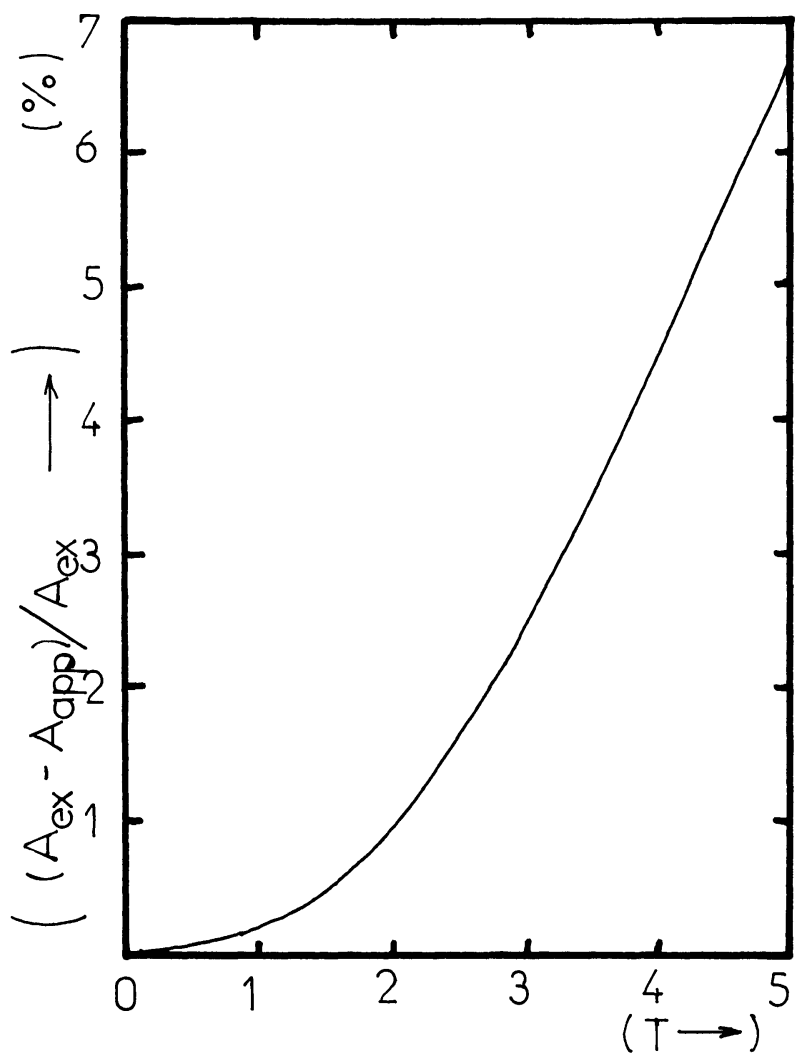

Fig. 1. - Erreur relative due à l'approximation de l'équation (3).

[Relative error due to the use of eq. (3).]

Il est probable que cette formule utilisée à la comparaison de deux intensités conduit à des erreurs moindres dans l'évaluation de leur rapport. Par ailleurs dans le cas d'un spectre à plusieurs raies c'est l'épaisseur relative à chaque raie : $C_{\mathrm{i}}^{(\mathrm{I}, \mathrm{II})} T$ qui intervient $\left(C_{\mathrm{i}}^{\mathrm{I}, \mathrm{II}}\right.$ définis plus loin).

Le but de cette note est d'abord de montrer qu'on peut généraliser l'équation (3) au cas des spectres Mössbauer à raies multiples (poudre ou monocristal) même si les raies sont dégénérées, pourvu qu'elles soient bien résolues ; ensuite d'exposer l'application de cette formule approchée à des spectres expérimentaux $\mathrm{du}$ fer métallique, à température ambiante, pour lesquels toutes les raies sont très bien résolues. Nous ne discutons pas ici des élargissements des raies dues à l'épaisseur effective, mais simplement de la variation des surfaces relatives des raies.

1. Absorbeur à raies multiples, effets de polarisation. - Nous ne considérons, par simplicité, que le cas où les raies Mössbauer sont bien résolues, et nous allons montrer qu'il existe, pour une raie donnée, des états propres de polarisation. Ce problème, posé depuis de nombreuses années [4] résulte du fait que les parties réelle (dispersion) et imaginaire (absorption) du tenseur de rang 2 qui représente l'indice de réfraction dans l'espace des états de polarisation pourraient avoir des vecteurs propres différents. Dans ce cas l'état de polarisation d'un rayonnement incident changerait progressivement au cours de la traversée de l'absorbeur, quel que soit l'état initial de la polarisation (le calcul de l'intensité transmise est alors compliqué [4c]).

Nous avons récemment montré [6] qu'il existait toujours, dans le cas du rayonnement dipolaire magnétique de ${ }^{57} \mathrm{Fe}$, des états propres de polarisation pour une raie d'absorption donnée (même dégénérée). En voici une démonstration directe :

Soient donc une source monochromatique située en $S$ et un absorbeur monocristallin situé en $A$ (Fig. 2). Choisissons la direction $S A$ (vecteur unitaire u) comme axe de quantification du système.

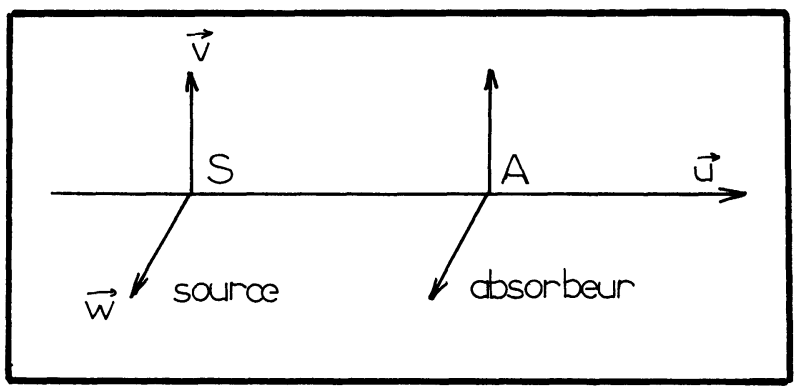

Fig. 2. - Système d'axes $\mathbf{v}, \mathbf{w}, \mathbf{u}$.

[Axis set $\mathbf{v}, \mathbf{w}, \mathbf{u}$.]

La source, non polarisée, peut être représentée par deux dipôles magnétiques, indépendants, égaux, tournant autour de u en sens opposés. Il en résulte des champs magnétiques $H^{ \pm}$, dont les composantes sont données par (notation complexe) :

$$
\left.\begin{array}{l}
H_{\mathrm{v}}^{ \pm}=-1 \\
H_{\mathrm{w}}^{ \pm}=\mp i
\end{array}\right\} \begin{gathered}
\text { champ rayonné vers } \mathbf{u}, \\
\text { composantes circulaires } \pm .
\end{gathered}
$$

L'interaction avec le moment magnétique $\mathbf{M}$ du noyau absorbeur s'écrit :

$$
\mathscr{H}_{\mathrm{int}}=-\mathbf{M} \cdot \mathbf{H}^{ \pm} \text {pour chacun des dipôles } .
$$

\section{Posons}

$$
\mathbf{M}=M_{\mathbf{u}} \mathbf{u}+M_{\mathbf{v}} \mathbf{v}+M_{\mathbf{w}} \mathbf{w}, \quad \mathbf{H}^{ \pm}=-\mathbf{v} \mp i \mathbf{w},
$$

il vient alors

$$
\mathscr{H}_{\mathrm{int}}^{( \pm)}=M_{\mathrm{v}} \pm i M_{\mathrm{w}} .
$$

On associe au moment dipolaire magnétique $\mathbf{M}$ 
un opérateur tensoriel irréductible d'ordre $1[7$, p. 491] dont les composantes standard [7, p. 489] sont :

$$
\begin{gathered}
M^{(+)}=-\frac{1}{\sqrt{2}}\left(M_{\mathrm{v}}+i M_{\mathrm{w}}\right) ; \quad M^{(0)}=M_{\mathrm{u}} ; \\
M^{(-)}=\frac{1}{\sqrt{2}}\left(M_{\mathrm{v}}-i M_{\mathrm{w}}\right),
\end{gathered}
$$

ce qui conduit à $\mathcal{H}^{( \pm)}=\mp \sqrt{2} M^{( \pm)}$.

Les éléments de matrice des opérateurs $M^{(1)}$, donnés dans le tableau I, se calculent par le théorème de Wigner-Eckart ; ils sont proportionnels aux coefficients de Clebsch-Gordan, soit, pour $M^{(+1,0,-1)}$ respectivement,

$$
\left\langle I_{g} 1 m_{g} \stackrel{1}{0}{ }_{1} \mid I_{e} m_{e}\right\rangle
$$

où $I_{g}, m_{g}$ décrivent l'état fondamental et $I_{e}, m_{e}$ l'état excité de la transition. Ces expressions permettent le calcul de l'indice de réfraction de l'absorbeur pour la raie considérée.

En effet l'indice de réfraction, pour une raie donnée, s'écrit d'après $[4 c]$ :

$$
n=1+\left(2 \pi N / k_{0}^{2}\right) \bar{f},
$$

où $\mathbf{k}_{0}$ est le vecteur d'onde du photon incident (et du photon diffusé), et $\bar{f}$ l'amplitude de diffusion cohérente, qui dépend de l'état de polarisation $(p) \mathrm{du}$ photon incident, ainsi que de celui $\left(p^{\prime}\right)$ du photon diffusé. $\bar{f}$ est donc un tenseur de rang 2, de composantes $\bar{f}_{p p^{\prime}}$. Il en est de même pour l'indice de réfraction [n], dont les quatre composantes complexes s'écrivent traditionnellement dans une base de polarisation circulaire :

$$
n_{++}, n_{--}, n_{+-}, n_{-+} \text {. }
$$

Les composantes de $\bar{f}$, pour une transition donnée, non dégénérée, s'écrivent, selon l'équation (11) de Blume [4c] :

$$
\begin{aligned}
\bar{f}_{p^{\prime} p}^{(i)}=\left(-\frac{k_{0} V R}{2 \pi h c}\right) \frac{1}{2 I_{g}+1} \times \\
\times \frac{\left\langle\mathbf{k}_{0} p^{\prime}, g|\mathcal{H}| e\right\rangle\left\langle e|\mathcal{H}| \mathbf{k}_{0} p, g\right\rangle}{E_{0}-E_{\mathrm{i}}+i \Gamma / 2}
\end{aligned}
$$

où

$E_{0}=$ énergie du rayonnement incident,

$E_{\mathrm{i}}=$ énergie de la transition,

$\mathscr{H}=$ interaction entre le moment magnétique nucléaire et le photon de polarisation considérée.

Appelons $\mathcal{A}_{p p^{\prime}}^{(\mathrm{i})}$ le numérateur de la dernière expression. Si l'on choisit pour $(p)$ et $\left(p^{\prime}\right)$ les états de polarisation circulaire déjà introduits, $\mathfrak{H}$ intervient par le terme $\mathfrak{H e}^{( \pm)}$selon que le photon considéré possède la polarisation ( \pm ). Un calcul simple, tenant compte des valeurs de $M^{( \pm)}$, indiquées dans le tableau I (valeurs réelles), donne le résultat suivant :

$$
\begin{aligned}
& \mathcal{A}_{++}^{(\mathrm{i})} \sim\left|\left\langle g\left|M^{(+)}\right| e\right\rangle\right|^{2}=\left\langle g\left|M^{(+)}\right| e\right\rangle\left\langle g^{*}\left|M^{(+)}\right| e^{*}\right\rangle \\
& \mathcal{A}_{-}^{(\mathrm{i})} \sim\left|\left\langle g\left|M^{(-)}\right| e\right\rangle\right|^{2}=\left\langle g\left|M^{(-)}\right| e\right\rangle\left\langle g^{*}\left|M^{(-)}\right| e^{*}\right\rangle \\
& \mathcal{A}_{+-}^{(\mathrm{i})} \sim\left\langle g\left|M^{(+)}\right| e\right\rangle\left\langle g^{*}\left|M^{(-)}\right| e^{*}\right\rangle \\
& \mathcal{A}_{-+}^{(\mathrm{i})} \sim\left\langle g\left|M^{(-)}\right| e\right\rangle\left\langle g^{*}\left|M^{(+)}\right| e^{*}\right\rangle=\mathcal{A}_{+-}^{(\mathrm{i}) *} .
\end{aligned}
$$

La matrice $\mathfrak{A}^{(\mathbf{i})}$ est hermitique, donc diagonalisable ; les tenseurs $[\bar{f}]$ et $[n]$ sont donc également diagonalisables. Dans le cas d'une raie non dégénérée, un calcul algébrique montre que, quels que soient $|e\rangle$ et $|g\rangle$, la matrice $\mathfrak{A}^{(\mathrm{i})}$ possède une valeur propre nulle. Cela correspond au résultat, en fait prévisible, qu'une raie non dégénérée d'un monocristal est entièrement polarisée.

Tableau I. - Eléments de matrice calculés lorsque l'axe de quantification est la direction de propagation $\mathbf{u}$ du rayonnement.

[Matrix elements (quantization axis : $\mathbf{u})$.]

\begin{tabular}{cccccc}
$|e\rangle$ & $|3 / 2\rangle$ & $|1 / 2\rangle$ & $|-1 / 2\rangle$ & $|-3 / 2\rangle$ & Matrice \\
- & - & - & - & - & - \\
$\langle g|=\langle 1 / 2|$ & 1 & 0 & 0 & 0 & $M^{(+)}$ \\
\hline$\langle-1 / 2|$ & 0 & $1 / \sqrt{3}$ & 0 & 0 & $M^{(0)}$ \\
\hline$\langle 1 / 2|$ & 0 & $\sqrt{2} / \sqrt{3}$ & 0 & 0 & $M^{(-)}$ \\
$\langle-1 / 2|$ & 0 & 0 & $\sqrt{2} / \sqrt{3}$ & 0 &
\end{tabular}


Lorsque la raie est dégénérée (plusieurs transitions différentes de même énergie), l'amplitude totale de diffusion résulte de la somme d'amplitudes partielles; il faut faire la somme de plusieurs matrices $\mathfrak{A}^{(\mathrm{i})}$, ce qui conduit encore à une matrice hermitique, donc diagonalisable.

Par conséquent $[\bar{f}]$ et $[n]$ sont toujours diagonalisables, et, pour une raie résolue, même dégénérée, il existe deux états propres de polarisation.

$\mathrm{Ce}$ résultat simplifie considérablement le calcul des effets d'épaisseur, si l'on choisit maintenant comme base de polarisation les états propres de la raie considérée. On peut en particulier définir des probabilités relatives d'absorption polarisées $C_{i}^{\mathrm{I}, \mathrm{II}}$, proportionnelles à la partie imaginaire de $[\bar{f}]$, donc aux valeurs propres de la matrice $\mathcal{A}^{(\mathbf{i})}$ associée à la raie considérée (la notation $C_{\mathrm{i}}^{\mathrm{I}, \mathrm{II}}$ représente les valeurs propres de la matrice $\rho_{\mathrm{q}^{\prime}}^{\mathrm{i}}$, définie par Gonser et coll. $\left.[4 b]\right)$.

Remarque 1. - Pour simplifier l'exposé du calcul, nous avons pris une base de quantification liée aux axes du rayonnement. Dans la pratique, $|e\rangle$ et $|g\rangle$ sont plutôt calculés dans les axes du gradient de champ électrique. Il y a alors lieu d'utiliser des matrices $\mathcal{M}^{(\text {) }}$, transmuées de $M^{(~)}$ par le changement d'axes, dont les valeurs sont données en appendice.

Remarque 2. - La probabilité relative d'absorption de la raie (i) avec une source non polarisée, dans un calcul sans effets d'épaisseur est simplement donnée par la trace de $\mathcal{A}^{(\mathbf{i})}$ :

$$
\begin{aligned}
I_{g \rightarrow e} \sim C_{\mathrm{i}}^{(\mathrm{I})}+C_{\mathrm{i}}^{(\mathrm{II})} \sim\left|\left\langle g\left|\mathcal{M}^{(+)}\right| e\right\rangle\right|^{2}+ \\
+\left|\left\langle g\left|\mathcal{M}^{(-)}\right| e\right\rangle\right|^{2} .
\end{aligned}
$$

La généralisation de cette expression au cas d'un absorbeur en poudre donne le résultat simple connu (pour laquelle le changement d'axes est inutile) :

$$
\begin{aligned}
\left|\left\langle g\left|M^{(+)}\right| e\right\rangle\right|^{2}+\left|\left\langle g\left|M^{(0)}\right| e\right\rangle\right|^{2}+ \\
+\left|\left\langle g\left|M^{(-)}\right| e\right\rangle\right|^{2} .
\end{aligned}
$$

2. Effets d'épaisseur. - Nous considérons une source monochromatique, non polarisée; le rayonnement émis peut être considéré comme la superposition de deux rayonnements d'égale intensité, polarisés suivant deux états orthogonaux. On choisit pour chaque raie d'absorption (i) ses deux états propres de polarisation, notés (I), (II), qui selon la symétrie pourront être soit rectilignes $(/ /)$ et $(\perp)$, soit circulaires $(+)$ et $(-)$, soit quelconques.

L'épaisseur effective associée à une raie (i), pour un rayonnement de polarisation $(\alpha)$ sera $T C_{i}^{(\alpha)}$; les $C_{i}^{(\alpha)}$ sont les probabilités relatives d'absorption polarisées, normalisées, selon [4], par $\sum_{\mathrm{i}} C_{\mathrm{i}}^{(\alpha)}=1$. La normalisation est donc la suivante :

$$
\sum_{\mathrm{i}}\left(C_{\mathrm{i}}^{(\mathrm{I})}+C_{\mathrm{i}}^{(\mathrm{II})}\right)=2
$$

(on remarquera qu'avec un absorbeur en poudre (non polarisé) on a $C_{\mathrm{i}}^{(\mathrm{I})}=C_{\mathrm{i}}^{(\mathrm{II})}$; on définit alors des coefficients non polarisés : $C_{\mathrm{i}}=\left(C_{\mathrm{i}}^{(\mathrm{I})}+C_{\mathrm{i}}^{(\text {III) }}\right) / 2$, tels que $\left.\sum_{\mathrm{i}} C_{\mathrm{i}}=1\right)$.

L'absorption se produit de manière indépendante pour les deux composantes de polarisation. La raie (i) aura donc une intensité relative d'absorption donnée par

$A_{\mathrm{i}}=A_{\mathrm{i}}^{(\mathrm{I})}+A_{\mathrm{i}}^{(\mathrm{II})} \sim T\left[\frac{C_{\mathrm{i}}^{(\mathrm{I})}}{1+C_{\mathrm{i}}^{(\mathrm{I})} T / 4}+\frac{C_{\mathrm{i}}^{(\mathrm{II})}}{1+C_{\mathrm{i}}^{(\mathrm{II})} T / 4}\right]$.

On remarquera que cette expression est égale, au facteur $T$ près, à celle que nous avons donnée pour les effets d'auto-absorption dans une source à raies multiples [5]; elle conduit donc à des intensités relatives identiques.

L'expérience donne des intensités relatives, que l'on peut comparer aux quantités normalisées

$$
A_{\mathrm{i}}{ }^{\prime}=A_{\mathrm{i}} / \sum_{\mathrm{i}} A_{\mathrm{i}} \cdot
$$

Nous présentons maintenant des expériences réalisées à température ambiante avec des absorbeurs de fer, et une source de ${ }^{57} \mathrm{Co}$ dans une matrice de rhodium, non polarisée.

3. Absorbeur de fer, entièrement polarisé. - Le premier absorbeur de fer utilisé (fabrication AMERSHAM, épaisseur $12,5 \mu \mathrm{m}$ fer naturel) a été placé dans un champ magnétique de $0,4 \mathrm{~T}$ fourni par un aimant permanent, la direction du champ étant perpendiculaire à la direction de propagation du rayonnement. Ensuite nous avons refait l'expérience avec deux lames superposées, puis quatre.

Dans toutes les expériences, le rapport $d / D$ (diamètre de la fenêtre du compteur/distance sourcecompteur) était de l'ordre de $1 / 10$; il en résulte une erreur d'étalonnage négligeable, $\mathrm{d} v / v \sim d^{2} / 16 D^{2}$ inférieure à $10^{-3}$, sans influence sur les intensités relatives des raies expérimentales (effet de cosine smearing [9]).

Les spectres obtenus, indiqués sur la figure 3, conduisent par affinement avec des raies Lorentziennes, à des intensités qui diffèrent sensiblement des valeurs attendues dans le cas d'un absorbeur mince (Tableau II).

Les probabilités d'absorption dans ce cas, sont les suivantes :

$$
\begin{array}{ll}
C_{1}^{\|}=C_{6}^{\|}=3 / 8, & C_{1}^{\perp}=C_{6}^{\perp}=0 ; \\
C_{2}^{\|}=C_{5}^{\|}=0, & C_{2}^{\perp}=C_{5}^{\perp}=1 / 2 ; \\
C_{3}^{\|}=C_{4}^{\|}=1 / 8, & C_{3}^{\perp}=C_{4}^{\perp}=0 .
\end{array}
$$

Nous avons calculé la variation des intensités $A_{\mathrm{i}}^{\prime}$ en fonction de $T$ en utilisant la formule exacte (éq. (2)) 


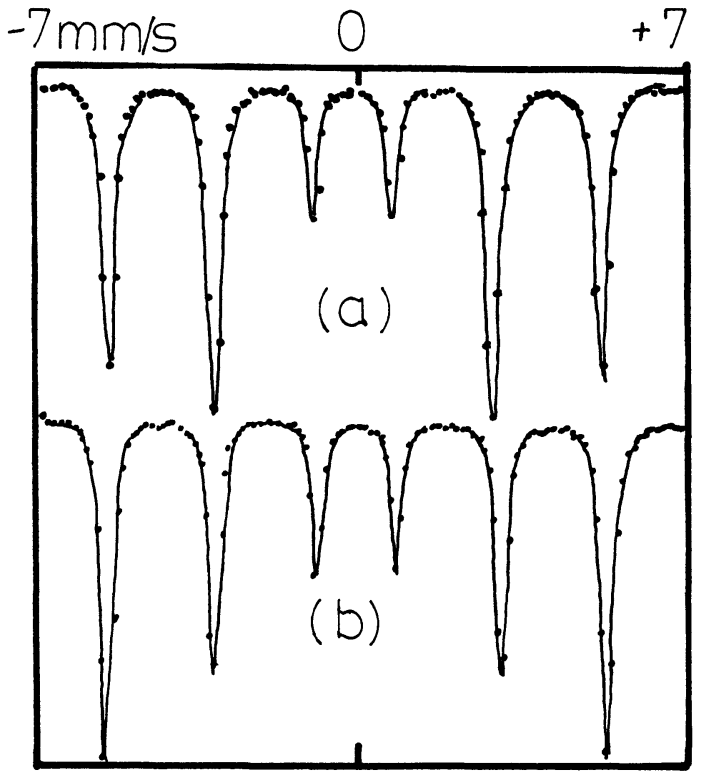

Fig. 3. - Spectres expérimentaux : lamelle de fer aimantée, épaisseur $50 \mu \mathrm{m}(a)$, absorbeur de fer en poudre, d'épaisseur $300 \mathrm{mg} / \Pi \mathrm{cm}^{2}(b)$. Les courbes tracées sont des Lorentziennes ajustées par paires symétriques.

[Experimental spectra : magnetized iron foil (a), powder absorber (b). Solid lines are Lorentzian lines fitted as symmetrical pairs.]

Tableau II. - Intensités relatives des raies Mössbauer, déduites des spectres expérimentaux par affinement à des raies Lorentziennes. On a indiqué entre parenthèses les valeurs théoriques prévues pour un absorbeur mince. Dans le cas de la poudre, ce sont les valeurs moyennes sur les deux séries d'échantillons qui sont données.

[Relative intensities of the Mössbauer lines (least square fit using Lorentzian lineshape). The thin absorber theoretical values are given for comparison.]

$\begin{array}{ccccc}\text { Absorbeur } & \begin{array}{c}\text { Epaisseur } \\ \text { (masse) }\end{array} & \begin{array}{c}\text { Raies 1,6 } \\ (\%)\end{array} & \begin{array}{c}\text { Raies 2,5 } \\ (\%)\end{array} & \begin{array}{c}\text { Raies 3,4 } \\ (\%)\end{array} \\ \text { Fer aimanté } & - & - & - & - \\ & 12,5 \mu \mathrm{m} & 37,8(37,5) & 46,6(50) & 15,6(12,5) \\ & 25 \mu \mathrm{m} & 37,6(37,5) & 44,9(50) & 17,5(12,5) \\ \text { Fer poudre } & 50 \mu \mathrm{m} & 37,0(37,5) & 43,9(50) & 19,1(12,5) \\ & (100 \mathrm{mg}) & 46,8(50) & 34,0(33,3) & 19,2(16,7) \\ & (200 \mathrm{mg}) & 45,3(50) & 34,0(33,3) & 20,7(16,7) \\ & (300 \mathrm{mg}) & 44,7(50) & 33,9(33,3) & 21,4(16,7)\end{array}$

et la formule approchée (éq. (3)). Les courbes correspondantes apparaissent figure 4 .

Les valeurs mesurées (Tableau II) conduisent aux résultats portés dans le tableau III. On constate sur

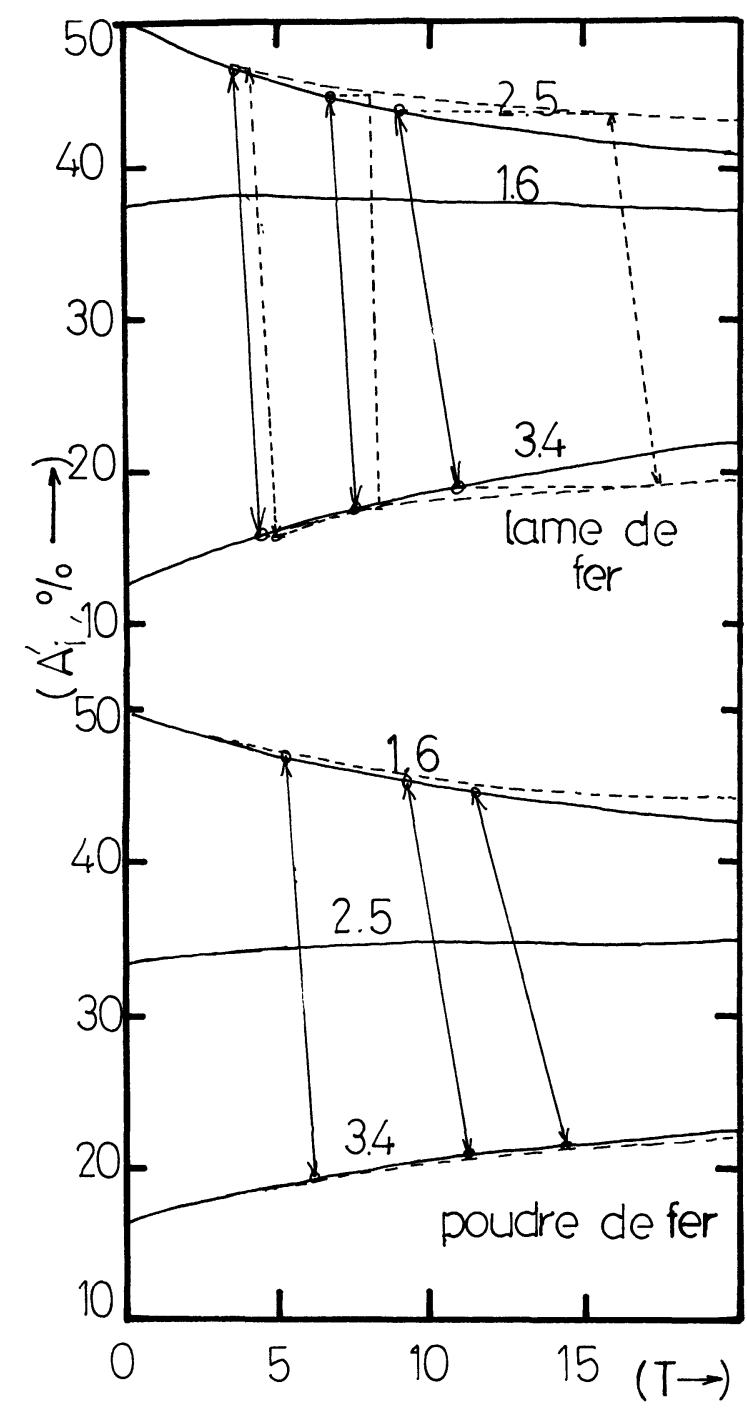

Fig. 4. - Intensités relatives calculées, et comparaison aux résultats expérimentaux du tableau I (trait plein : formule approchée; pointillés : formule exacte).

[Calculated relative intensities, compared with the data of figure 1 (full line : approximate formula, dotted line : actual formula).]

Tableau III. - Valeurs de $T$ et $f$, lame de fer aimantée.

[Values of $T$ and $f$, magnetized iron foil.]

Epaisseur absorbeur (en $\mu \mathrm{m}$ )

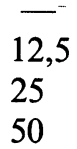

Formule approchée (éq. (3))

$\begin{array}{cc}\frac{T}{-} & f \\ 4 \pm 0,2 & 0,70 \pm 0,03 \\ 7,1 \pm 0,4 & 0,62 \pm 0,03 \\ 10 \pm 1 & 0,44 \pm 0,03\end{array}$

Formule exacte (éq. (2))

$\begin{array}{cc}T & \frac{f}{-} \\ 4,5 \pm 0,2 & 0,78 \pm 0,03 \\ 8,2 \pm 0,4 & 0,71 \pm 0,03 \\ 16,8 \pm 1 & 0,73 \pm 0,03\end{array}$


ce tableau que les valeurs de $f$ obtenues sont en accord avec celles de la littérature $(0,80,0,81)$ [10] lorsque l'on tend vers l'absorbeur mince.

4. Absorbeur de fer en poudre. - Nous avons réalisé deux séries de trois absorbeurs avec de la poudre de fer naturel, de masse $100,200,300 \mathrm{mg}$ répartie sur $\Pi \mathrm{cm}^{2}$.

Pour tenter d'obtenir une dispersion homogène du fer nous avons confectionné nos absorbeurs selon 2 méthodes différentes : mélange avec du nitrure de bore ( $1^{\text {re }}$ série) et avec de la résine époxyde ( $2^{\mathrm{e}}$ série). Nous avons également mesuré au microscope la taille des grains : de 3 à $5 \mu \mathrm{m}$. Dans le cas de l'absorbeur le plus mince, l'épaisseur moyenne de la couche équivalente de fer, $40 \mu \mathrm{m}$, correspond à un nombre suffisant de grains pour admettre en première approximation que cet absorbeur peut se présenter de manière homogène (et $a$ fortiori pour les absorbeurs plus épais).

Les spectres obtenus (Fig. $3 b$ ), très semblables pour les deux séries, conduisent à des intensités de raies qui diffèrent de plus en plus des valeurs attendues dans le cas d'un absorbeur mince (Tableau II $b$ ).

Les probabilités d'absorption, égales pour les deux états polarisés de base, sont :

$$
\begin{aligned}
& C_{1}^{(1)}=C_{2}^{(2)}=C_{6}^{(1)}=C_{6}^{(2)}=1 / 4 \\
& C_{2}^{(1)}=C_{2}^{(2)}=C_{5}^{(1)}=C_{5}^{(2)}=1 / 6 \quad\left(\sum_{\mathrm{i}} C_{\mathrm{i}}^{(\alpha)}=1\right) \\
& C_{3}^{(1)}=C_{3}^{(2)}=C_{4}^{(1)}=C_{4}^{(2)}=1 / 2 .
\end{aligned}
$$

Comme précédemment nous avons tracé (Fig. 4) la variation des intensités relatives en fonction de $T$ en utilisant la formule exacte (éq. (2)) et la formule approchée (éq. (3)).

Les valeurs mesurées (Tableau II) nous permettent de déduire les valeurs de $T$ et de $f$ correspondant à chacun des absorbeurs en poudre (Tableau IV).

On remarquera le bon accord entre les intensités mesurées et calculées des raies 2 et 5 : respectivement 34,0 et $33,8 \%$, cette dernière valeur étant pratiquement indépendante de $T$. Un résultat contraire impliquerait une certaine texture (voir par exemple [5]). Nous disposons donc d'un bon test de l'isotropie de l'absorbeur.
5. Discussion. - La figure 5 fait apparaître les déterminations expérimentales de $T$ et $T / m$ en fonction de $m$ (= masse de l'échantillon).

Nous discutons d'abord les résultats de la lame de fer aimantée (Fig. 5a), qui sont bons puisque la

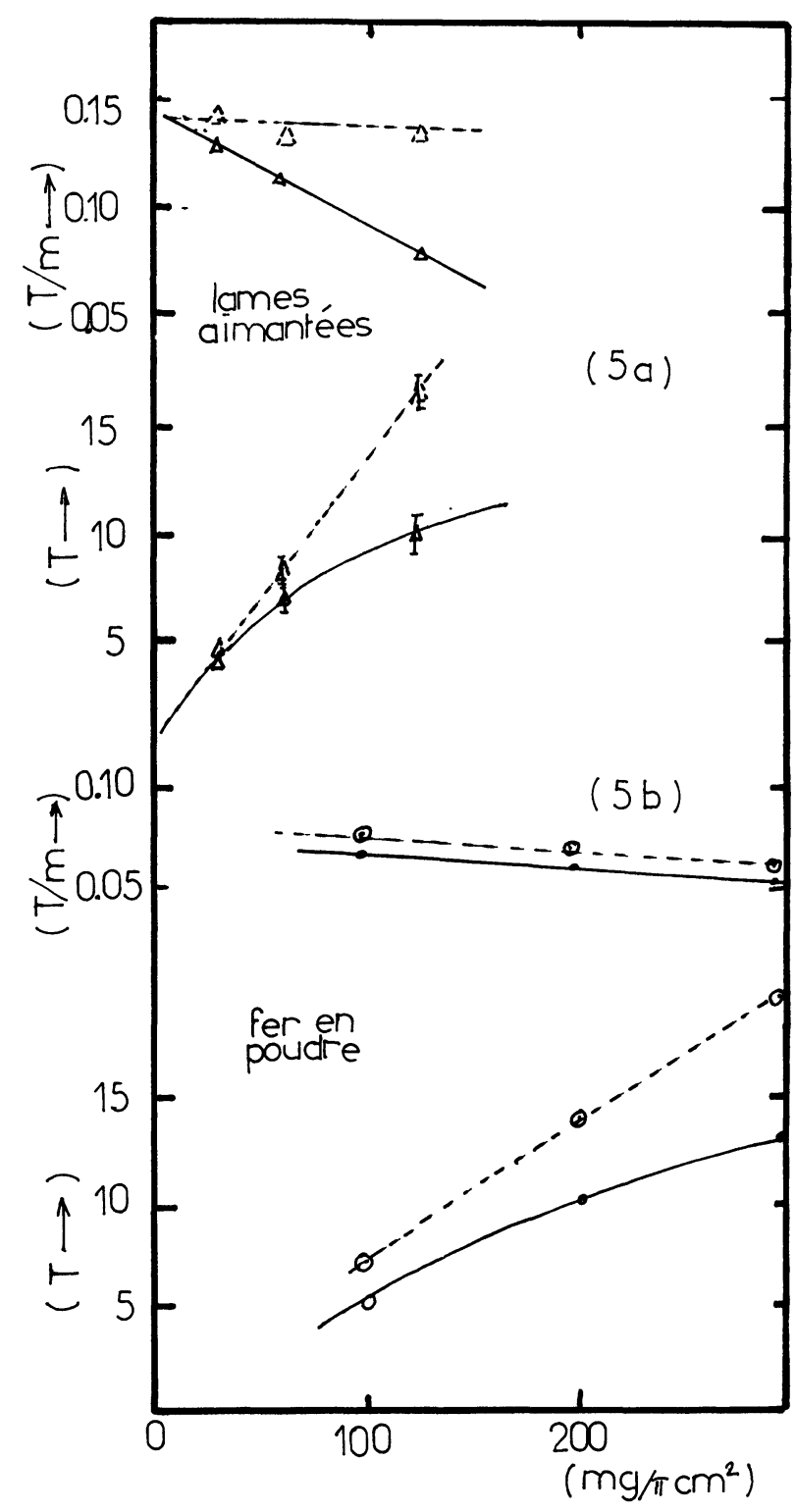

Fig. 5. - Valeurs de $T$ et $T / m$ en fonction de $m$ (masse de fer par $\Pi \mathrm{cm}^{2}$ ) pour les divers échantillons.

[Values of $T$ and $T / m\left(m=\right.$ weight of iron per $\left.\Pi \mathrm{cm}^{2}\right)$.]

Tableau IV. - Valeurs de T et $f$, fer en poudre $\left(\Pi \mathrm{cm}^{2}\right)$.

[Values of $T$ and $f$, powdered iron.]

\begin{tabular}{ccc}
$\begin{array}{c}\text { Masse absorbeur } \\
(\mathrm{mg})\end{array}$ & \multicolumn{2}{c}{ Formule approchée (éq. (3)) } \\
- & $T$ & $f$ \\
100 & - & - \\
200 & $5,5 \pm 0,5$ & $0,30 \pm 0,03$ \\
300 & $10 \pm 1$ & $0,28 \pm 0,03$ \\
& $13 \pm 1,5$ & $0,24 \pm 0,03$
\end{tabular}

Formule exacte (éq. (2))

$\begin{array}{cc}\frac{T}{-} & - \\ 6,5 \pm 0,5 & 0,36 \pm 0,03 \\ 12 \pm 1 & 0,33 \pm 0,03 \\ 15 \pm 1,5 & 0,28 \pm 0,03\end{array}$


valeur obtenue pour $f$ avec la formule exacte (éq. (2)) est correcte $(0,78 \pm 0,04)$.

La valeur de $T / m$ obtenue par extrapolation à $m=0$, à partir des valeurs déterminées en utilisant la formule approchée, correspond à celle donnée par la formule exacte (éq. (2)).

L'usage de la formule approchée conduit, pour $m$ non nul, à un facteur $f$ trop petit ; c'est-à-dire que pour un $f$ donné cette méthode majore la correction des effets d'épaisseur (ex. : $T=4$ majore de $10 \%$, erreur que l'on peut corriger par extrapolation).

Dans le cas de la poudre les valeurs de $T / m$ conduisent à un facteur $f$ incorrect $(0,36 \pm 0,03)$. L'erreur due à l'approximation proposée est alors masquée par des phénomènes liés à ce type d'absorbeur tels que les élargissements des raies. Deux cas sont envisageables :

a) Un élargissement homogène des raies de l'absorbeur; dans ce cas la définition de l'épaisseur effective [1], $T=f a n \sigma_{0} t \Gamma_{0} / \Gamma$, conduirait bien à augmenter les valeurs de $f$ déduites de l'expérience (il faudrait aussi remplacer l'équation (3) par l'intégrale de transmission définie en [1]).

b) Un élargissement inhomogène dû à un effet de distribution de champ effectif vu par les noyaux. Celui-ci élargit les raies d'autant plus qu'elles sont éloignées du centre du spectre : les raies éloignées sont alors moins affectées par les effets de saturation, ce qui conduit effectivement d'après la figure 4 à une sous-estimation de $T$ : c'est-à-dire majoration des corrections d'épaisseur quand on travaille à $f$ donné. L'origine de cette distribution pourrait être le champ démagnétisant qui varie selon la forme et l'éloignement des grains $\left(^{*}\right)$ ou les inhomogénéités du maté- riau ; on s'attend à ce que ces effets soient tous deux négligeables dans une lame aimantée à saturation, et qui a subi un traitement métallurgique (recuit).

6. Conclusion. - Il ne s'agissait pas pour nous de mesurer une nouvelle fois la valeur du facteur $f$ dans le fer métallique, mais simplement de tester une formule simple d'évaluation des effets d'épaisseur.

Les résultats sont satisfaisants lorsque l'état cristallin est bon. L'erreur introduite par l'usage d'une formule plus simple que celle utilisant les fonctions de Bessel est alors décelable : il est possible d'estimer cette erreur et de la corriger par extrapolation. On constate que la correction d'épaisseur est toujours légèrement trop importante $(10 \%$ pour $T=4)$.

Par contre, lorsque l'état cristallin imparfait conduit à des élargissements inhomogènes des raies, cette erreur est dépassée par l'usage abusif d'une formule qui suppose des largeurs égales pour la source et l'absorbeur. L'utilisation de la méthode de correction proposée en supposant connue la valeur du facteur $f$ conduit à des corrections qui peuvent être nettement trop grandes, par exemple d'un facteur 2 dans notre échantillon de fer en poudre. Cette méthode de correction a été introduite dans nos programmes d'ajustement et est actuellement à l'essai pour la détermination complète du gradient de champ électrique d'un cristal épais.

Appendice. - Matrices transmuées $\mathcal{M}^{(1)}$.

Rappelons d'abord que les matrices $\left.M^{(}\right)$sont définies dans les axes du rayonnement (vwu). Les valeurs moyennes intervenant dans le calcul des probabilités de transition s'écrivent donc :

$$
\begin{aligned}
\left\langle g\left|M^{(1)}\right| e\right\rangle & =\left\langle\Phi_{v w u}^{(g)}\left|M^{(1)}\right| \Phi_{v w u}^{(e)}\right\rangle \\
& =\left\langle\Phi_{x y z}^{(g)}\left|R_{\alpha \beta \gamma}^{(1 / 2)} M^{(1)}\left(R_{\alpha \beta \gamma}^{(3 / 2)}\right)^{-1}\right| \Phi_{x y z}^{(e)}\right\rangle \\
& =\left\langle\Phi_{x y z}^{(g)}\left|\mathcal{M}_{\alpha \beta \gamma}^{(\beta)}\right| \Phi_{x y z}^{(e)}\right\rangle
\end{aligned}
$$

où $x y z$ sont les axes principaux du gradient de champ électrique (axes usuels) et $R_{\alpha \beta \gamma}$ des matrices de rotation ([7], p. 918) pour les angles d'Euler $\alpha \beta \gamma$ (voir Fig. 6).

$\left({ }^{*}\right)$ En ce qui concerne le problème de l'éloignement des grains, qui a été soulevé très récemment [11], il n'y a pas encore de résultats expérimentaux assez précis.

Fig. 6. - Orientation relative des axes du gradient $(x y z)$ et du rayonnement $(v w u) . \alpha=(O Y, O t) ; \beta=(O Z, O u) ; \gamma=(O t, O w)$; $\beta, \alpha$ sont les angles polaires de $\mathbf{u}$ dans $x y z$. (Ot est perpendiculaire à $O Z, O u$.)

[Relative orientation of the gradient axes $(x y z)$ and radiation axes $(v w u)$; do notice that $\beta, \alpha$ are the polar axes for $\mathbf{u}$ in the $x y z$ basis. $(O t$ is perpendicular to $O Z, O u$.)]

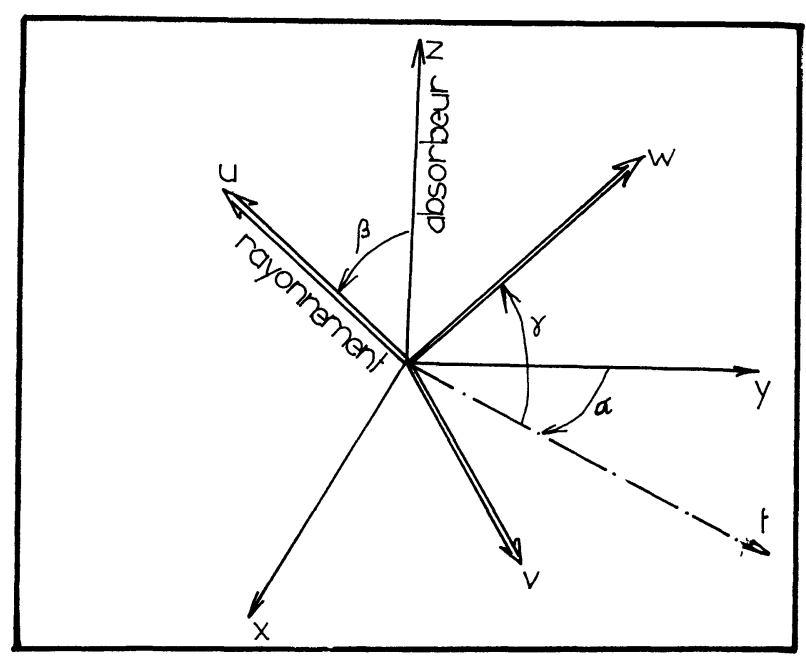


Le calcul des éléments des matrices $\mathcal{M}_{\alpha \beta \gamma}^{()}$se fait simplement $[6,8]$, et donne les résultats suivants :

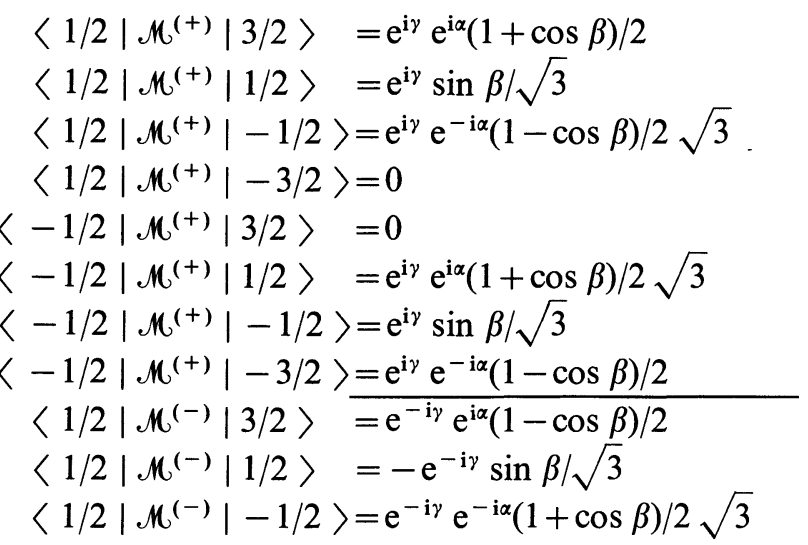

$$
\begin{aligned}
& \left\langle 1 / 2\left|\mathcal{M}^{(-)}\right|-3 / 2\right\rangle=0 \\
& \left\langle-1 / 2\left|\mathcal{M}^{(-)}\right| 3 / 2\right\rangle=0 \\
& \left\langle-1 / 2\left|\mathcal{M}^{(-)}\right| 1 / 2\right\rangle=\mathrm{e}^{-\mathrm{i} \gamma} \mathrm{e}^{\mathrm{i} \alpha}(1-\cos \beta) / 2 \sqrt{3} \\
& \left\langle-1 / 2\left|\mathcal{M}^{(-)}\right|-1 / 2\right\rangle=-\mathrm{e}^{-\mathrm{i} \gamma} \sin \beta / \sqrt{3} \\
& \left\langle-1 / 2\left|\mathcal{M}^{(-)}\right|-3 / 2\right\rangle=\mathrm{e}^{-\mathrm{i} \gamma} \mathrm{e}^{-\mathrm{i} \alpha}(1+\cos \beta) / 2 \\
& \left\langle 1 / 2\left|\mathcal{M}^{(0)}\right| 3 / 2\right\rangle=-\mathrm{e}^{\mathrm{i} \alpha} \sin \beta / \sqrt{2} \\
& \left\langle 1 / 2\left|\mathcal{M}^{(0)}\right| 1 / 2\right\rangle=\sqrt{2} \cos \beta / \sqrt{3} \\
& \left\langle 1 / 2\left|\mathcal{M}^{(0)}\right|-1 / 2\right\rangle=\mathrm{e}^{-\mathrm{i} \alpha} \sin \beta / \sqrt{6} \\
& \left\langle 1 / 2\left|\mathcal{M}^{(0)}\right|-3 / 2\right\rangle=0 \\
& \left\langle-1 / 2\left|\mathcal{M}^{(0)}\right| 3 / 2\right\rangle=0 \\
& \left\langle-1 / 2\left|\mathscr{M}^{(0)}\right| 1 / 2\right\rangle=-\mathrm{e}^{\mathrm{i} \alpha} \sin \beta / \sqrt{6} \\
& \left\langle-1 / 2\left|\mathscr{M}^{(0)}\right|-1 / 2\right\rangle=\sqrt{2} \cos \beta / \sqrt{3} \\
& \left\langle-1 / 2\left|\mathcal{M}^{(0)}\right|-3 / 2\right\rangle=\mathrm{e}^{-\mathrm{i} \alpha} \sin \beta / \sqrt{2}
\end{aligned}
$$

\section{Bibliographie}

[1] Margulies, S., Ehrman, J. R., Nucl. Instr. Methods 12 (1961) 131.

[2] LaNG, G., Nucl. Instr. Methods 24 (1963) 425.

[3] Article de revue et références :

SHENOY, G. K., FRIEDT, J. M., Mössbauer Effect Methodology 9 (1974) 277. Voir aussi :

GibB, T. C., Greatrex, R., Greenwood, N. N., J. Chem. Soc. A (1968) 890.

[4a] Byxov, G. A. et al., Soviet Phys. JETP 16 (1963) 646.

[4b] Housley, R. M., Grant, R. W., Gonser, V., Phys. Rev. 178 (1969) 514 [4c] Blume, M., Kistner, O. C., Phys. Rev. 171 (1968) 417.

[5] Henry, M., Varret, F., Revue Physs. Appl. 14 (1979) 289.

[6] Henry, M., (Thèse de 3 e cycle, Le Mans 1979).

[7] Messiah, A., Mécanique Quantique, Dunod, Paris.

[8] VARret, F., Thèse, Saclay 1972.

[9] An introduction to Mössbauer Spectroscopy, page 28 (Editor L. May, Plenum Press, New York, London) (1971).

[10] Trooster, J. M., Viegers, M. P. A., Mössbauer Effect Reference and Data Journal 1 (1978) 154.

[11] Van Der Woude, F., Int. Conf. Appl. Mössbauer Effect Portoroz (1979). 